\title{
CO-CO Coupling on Cu Facets: Coverage, Strain and Field Effects
}

Robert B. Sandberg, ${ }^{a}$ Joseph H. Montoya, ${ }^{\mathrm{a}}$ Karen Chan, ${ }^{\mathrm{a}, \mathrm{b}}$ Jens K. Nørskov ${ }^{*}, \mathrm{a}, \mathrm{b}$

${ }^{a}$ SUNCAT Center for Interface Science and Catalysis, Department of

Chemical Engineering, Stanford University, Stanford, CA 94305-5025, USA

${ }^{\mathrm{b}}$ SUNCAT Center for Interface Science and Catalysis, SLAC National Accelerator Laboratory, Menlo Park, CA 94025, USA

Email:norskov@stanford.edu 


\begin{abstract}
We present a DFT study on the effect of coverage, strain, and electric field on CO-CO coupling energetics on $\mathrm{Cu}$ (100), (111), and (211). Our calculations indicate that $\mathrm{CO}-\mathrm{CO}$ coupling is facile on all three facets in the presence of a cation-induced electric field in the Helmholtz plane, with the lowest barrier on $\mathrm{Cu}(100)$. The $\mathrm{CO}$ dimerization pathway is therefore expected to play a role in $\mathrm{C}_{2}$ formation at potentials negative of the $\mathrm{Cu}$ potential of zero charge, corresponding to $\mathrm{CO}_{2} / \mathrm{CO}$ reduction conditions at high $\mathrm{pH}$. Both increased ${ }^{*} \mathrm{CO}$ coverage and tensile strain further improve $\mathrm{C}-\mathrm{C}$ coupling energetics on $\mathrm{Cu}$ (111) and (211). Since CO dimerization is facile on all $3 \mathrm{Cu}$ facets, subsequent surface hydrogenation steps may also play an important role in determining the overall activity towards $\mathrm{C}_{2}$ products. Adsorption of $* \mathrm{CO}, * \mathrm{H}$, and $* \mathrm{OH}$ on the 3 facets were investigated with a Pourbaix analysis. The (211) facet has the largest propensity to coadsorb $* \mathrm{CO}$ and $* \mathrm{H}$, which would favor surface hydrogenation following $\mathrm{CO}$ dimerization.
\end{abstract}

\title{
Graphical Abstract
}

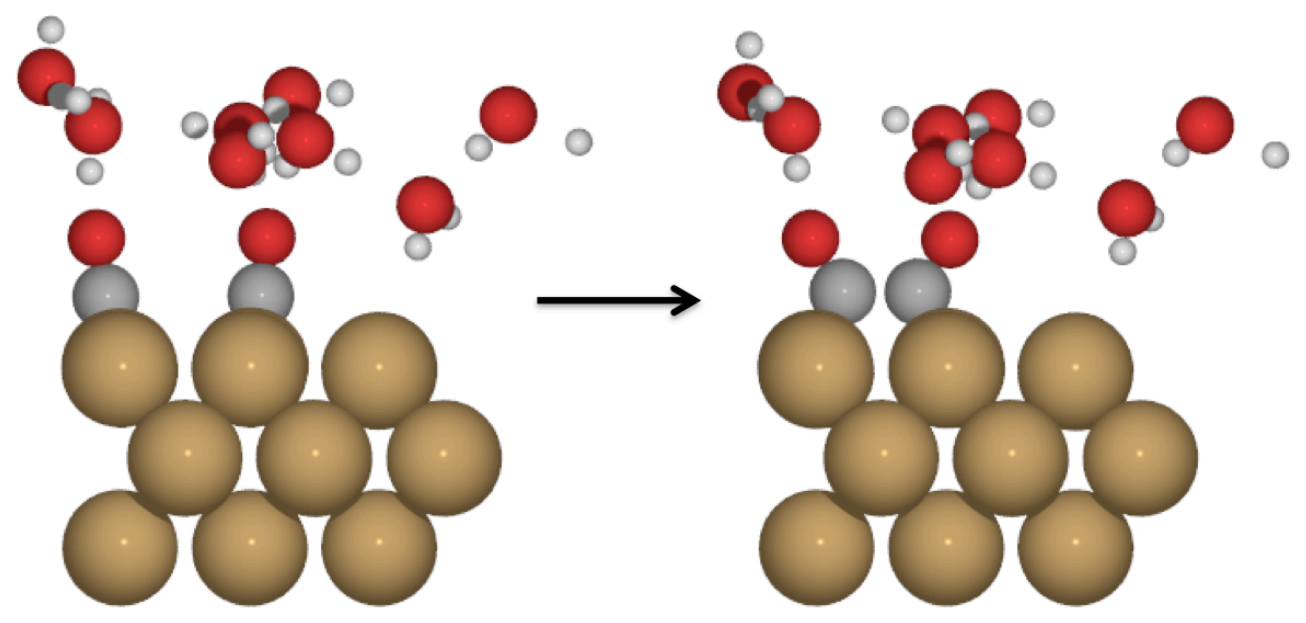

\section{Keywords}

Electrocatalysis, $\mathrm{CO}_{2}$ reduction, $\mathrm{C}-\mathrm{C}$ coupling, $\mathrm{CO}$ dimerization

\section{INTRODUCTION}

The electrochemical $\mathrm{CO}_{2}$ reduction reaction $\left(\mathrm{CO}_{2} \mathrm{RR}\right)$ has the potential to produce industrial chemicals and to mitigate the increasing $\mathrm{CO}_{2}$ concentrations in the atmosphere. Renewable energy sources such as wind and solar have seen great increases in efficiency in recent years. However, they are intermittent and require significant energy storage.

$\mathrm{CO}_{2} \mathrm{RR}$ has the potential to store renewable electricity in the form of energy-dense fuels. 
The demand for hydrocarbons and alcohols is immense, with an annual production rate on the order of $10^{13} \mathrm{~kg} / \mathrm{yr}$, or $1000 \mathrm{~kg}$ of production, on average, per person.[1] The two major challenges in $\mathrm{CO}_{2} \mathrm{RR}$ are the low efficiency and selectivity of known catalysts. Polycrystalline copper, the only transition metal catalyst to produce hydrocarbons at reasonable Faradaic efficiency, produces a reasonable $1 \mathrm{~mA} / \mathrm{cm}^{2}$ current density at $\sim 1 \mathrm{~V}$ overpotential.[2]

Reduction towards $\mathrm{C}_{2}$ hydrocarbons and alcohols such as ethylene, an industrial building block, and ethanol, a valuable fuel, is particularly desirable due to their higher energy densities than single carbon products. Polycrystalline $\mathrm{Cu}$ has shown similar onset potentials for $\mathrm{C}_{1}$ and $\mathrm{C}_{2}$ hydrocarbons at $-0.75 \mathrm{~V}$ vs. RHE.[3] These results are consistent with calculations which showed C-C coupling to be feasible after CO is hydrogenated,[4] which suggests both $C_{1}$ and $C_{2}$ pathways to be limited by the initial hydrogenation of ${ }^{*} \mathrm{CO}$ for form ${ }^{*} \mathrm{CHO}$. However, $\mathrm{C}_{2}$ products have been observed at low overpotential in alkaline conditions on the $\mathrm{Cu}(100)$ facet[5] and with oxide-derived copper (OD$\mathrm{Cu}$ ).[6,7] These results suggest an alternative C-C coupling pathway that proceeds prior to * $\mathrm{CO}$ hydrogenation. The $\mathrm{CO}$ dimerization mechanism had been proposed by Gattrell and Gupta et al.[8] and was then incorporated into a $\mathrm{CO}_{2}$ reduction mechanism.[9] The mechanism was then refined to include a solvent-stabilized *OCCO intermediate.[10] Recent calculations have found CO-CO coupling to be feasible in the presence of a solvent and cation, which induces a field in the Helmholtz plane.[11] This pathways would depend on cation concentration at the interface, which depends on the absolute, not RHE potential, and is therefore expected to depend on $\mathrm{pH}$. The $\mathrm{pH}$ of the electrolyte, and more importantly the local $\mathrm{pH}$ at the electrode | electrolyte interface, has been shown to influence the distribution of $C_{1}$ and $C_{2}$ products.[7,12,13] In this paper, we extend previous theoretical studies to the stepped (211) surface and examine the effects of coverage, strain, and field on *CO dimerization energetics.

\section{RESULTS}

\section{CO-CO coupling barriers}

Figure 1 shows the calculated electronic energy barriers for CO dimerization on $\mathrm{Cu}$ (100), (111), and (211) in the presence of a proton in the Helmholtz plane. Although this pathway is expected to play a role in alkaline media, it is quite difficult to fully solvate hydroxide in a water layer with DFT. Therefore, a proton was used to estimate the alkaline barrier; Montoya et al. found the stabilization of a proton to be equivalent to other cations.[11] A barrier of $0.45 \mathrm{eV}$ was found for $\mathrm{Cu}(100)$ and a barrier of $0.72 \mathrm{eV}$ was found for both $\mathrm{Cu}$ (111) and (211). All three of these barriers are lower than $0.75 \mathrm{eV}$, corresponding to a TOF of $1 \mathrm{~s}^{-1}$ at room temperature from transition state theory, which is considered to be a threshold for fast kinetics. The $\mathrm{CO}$ dimerization pathway is therefore expected to play a role in $\mathrm{C}_{2}$ formation at absolute potentials negative of the $\mathrm{Cu}$ potential of zero charge $\left(-0.7 \mathrm{~V}\right.$ vs. SHE),[14] corresponding to $\mathrm{CO}_{2} / \mathrm{CO}$ reduction at low overpotential in alkaline media.

The corresponding work function shifts along the reaction coordinate are shown in the bottom panels. Work function changes along the coupling pathway arise due to the shift in adsorbate dipole and slight changes in water structure. Assuming an SHE work 
function of 4.4eV,[15] and from $U_{S H E}=\frac{\Phi-\Phi_{S H E}}{e}$, these calculations have been performed at $\Phi \approx-1$ to $0 \mathrm{eV}$ vs SHE. Constant potential barriers can in principle be extrapolated using a computationally intensive cell-extrapolation scheme.[16] However, the key effect captured here is the cation-induced field stabilization of *OCCO, which depends on the ion distribution at the interface, and not directly on the chemical potential of electrons.[17]
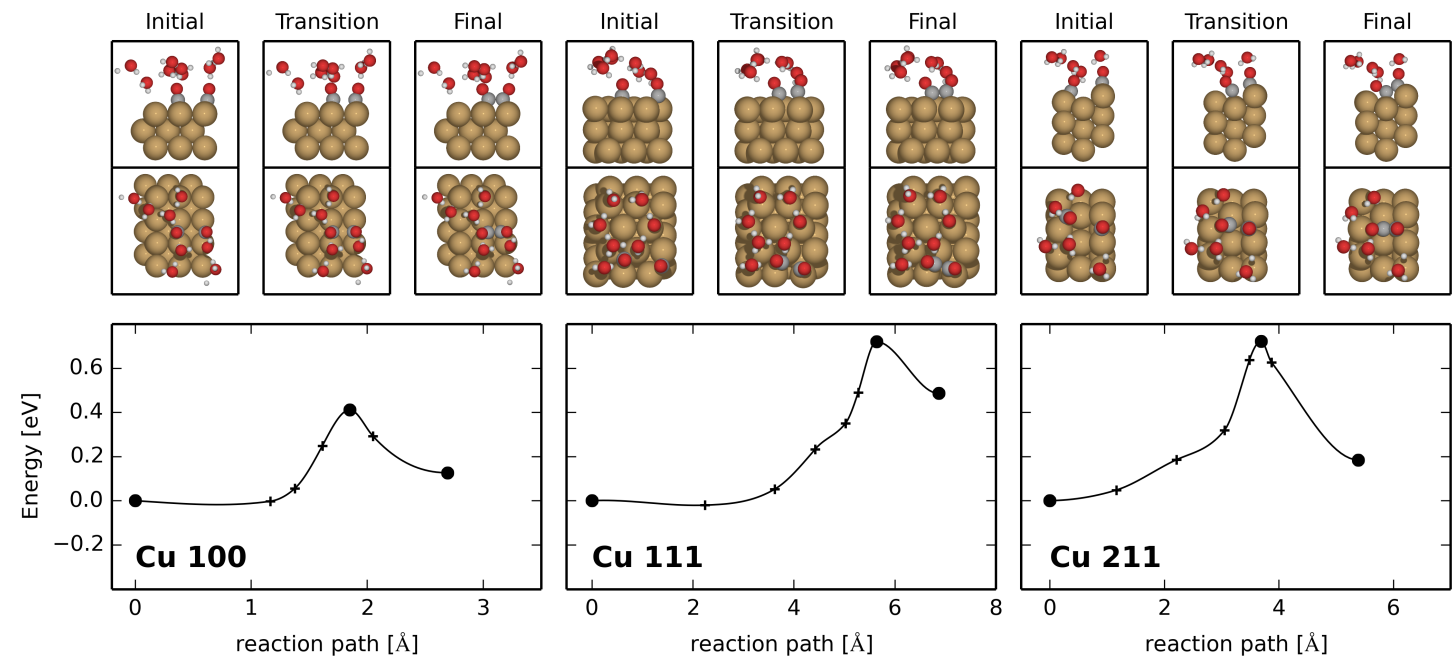

Figure 1. Structures and barriers for CO dimerization.

Field effects in catalysis have been studied for decades, such as in the case of alkali promoters in ammonia synthesis.[18] The change in energy of an adsorbate in an electric field is given by:

$$
\Lambda E=\mu \varepsilon-\frac{1}{2} \alpha \varepsilon^{2}+\ldots
$$

where $\Delta E$ represents the change in potential energy, $\varepsilon$ the field strength, $\mu$ the dipole moment, and $\alpha$ the polarizability. Figures 2 and 3 show the effect of an applied field on $\mathrm{CO}-\mathrm{CO}$ coupling as calculated via an applied sawtooth potential. Figure 2 shows the effect of field on the binding energies of the initial $\left(2{ }^{*} \mathrm{CO}\right)$, transition $\left({ }^{*} \mathrm{OC}-\mathrm{CO}\right)$, and final $\left({ }^{*} \mathrm{OCCO}\right)$ states on $\mathrm{Cu}(100)$, and the fitted dipole moments and polarizabilities. Across all three adsorbed species, the polarizabilities are very similar; however, the dipole moment changes significantly along the reaction coordinate. This shift in dipole moment of the * $\mathrm{CO}$ dimer relative to uncoupled ${ }^{*} \mathrm{CO}$ gives rise to the field stabilization of the transition and final states. Figure 3 shows the reaction energy as a function of the applied field. All three facets show a linear dependence on field, which arises from the differences in dipole moment between $2 * \mathrm{CO}$ and ${ }^{*} \mathrm{OCCO}$. 


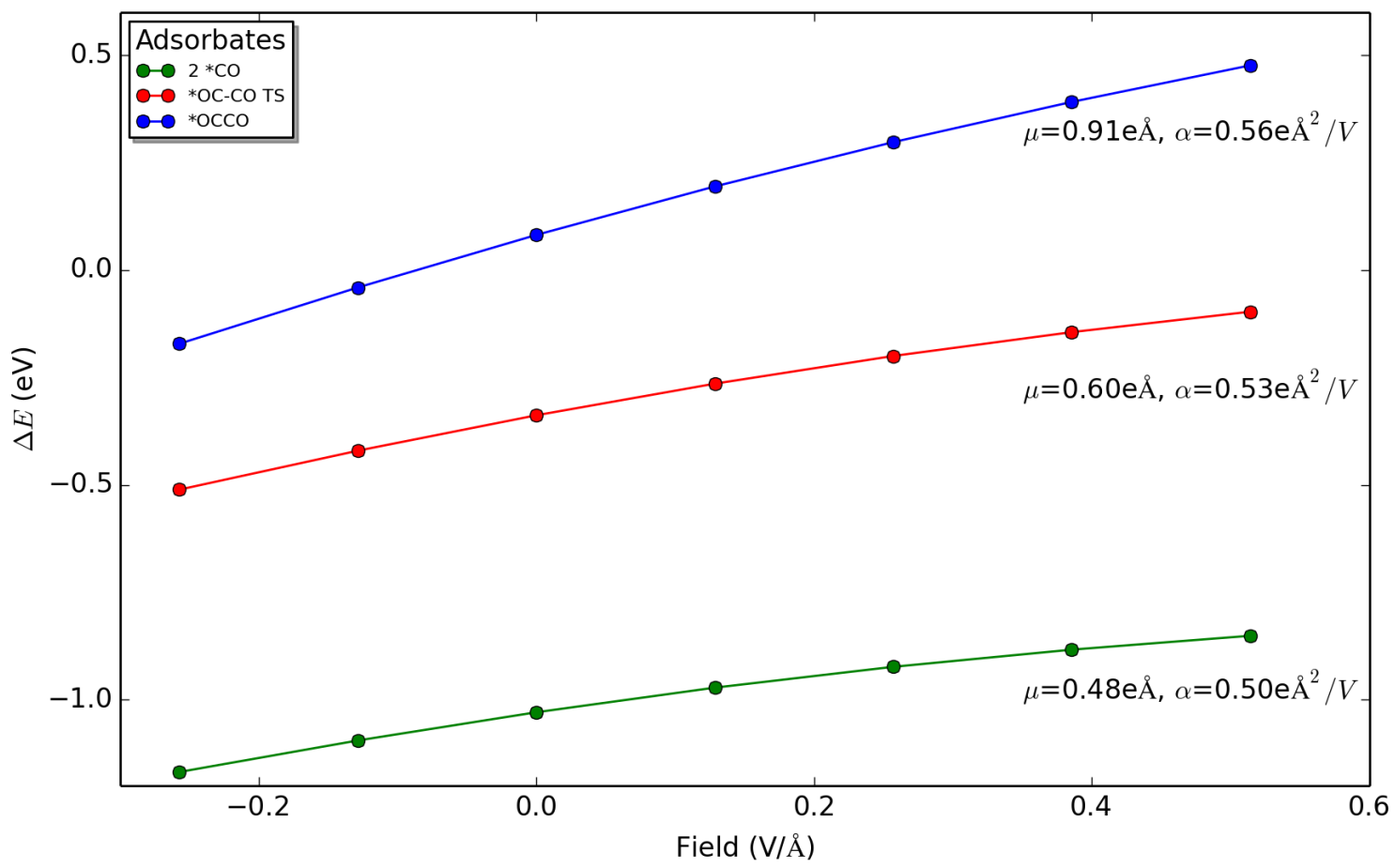

Figure 2. Adsorption energy as a function of field for CO-CO coupling adsorbates on $\mathrm{Cu}(100)$.

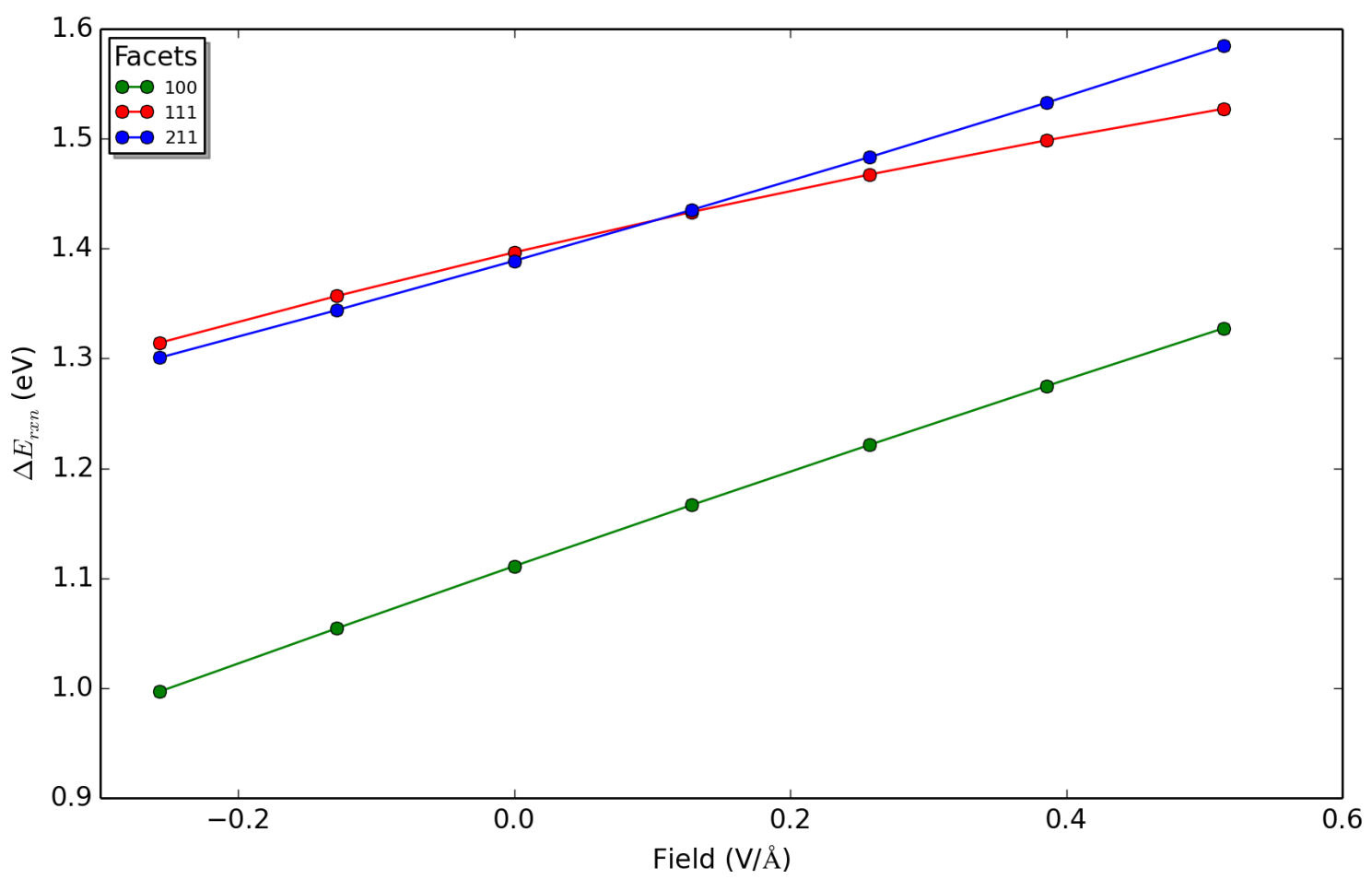

Figure 3. Reaction energy as a function of field for CO-CO coupling.

Previous work has suggested that ${ }^{*} \mathrm{CO}$ dimerization proceeds exclusively on the (100) facet.[4,5] While indeed the vacuum *OCCO binding energy is lowest on (100), 
*CO-*CO coupling barriers are surmountable on all 3 facets in the vicinity of an ion. Furthermore, the stability of the (100) facet under reducing conditions remains an open question. Surface energies of bare $\mathrm{Cu}$ facets suggest the predominance of (111) facets to (100).[19] In different electrochemical environments, $\mathrm{Cu}$ has been reported to reconstruct from (100) to (011) stripes[20] and from polycrystalline Cu to (100).[21]

Coverage effects

Adsorbate-adsorbate interactions have been shown to have significant impact on reaction energetics.[22] For transition metals, *CO binding energies significantly decrease with *CO coverage, which shifts the strong * $\mathrm{CO}$ binding metals along the theoretical activity volcano for $\mathrm{CO}_{2}$ reduction.[23] As discussed in the following section, $\mathrm{Cu}$ facets considered have calculated *CO coverage of up to 0.4 under equilibrium conditions at reducing conditions below $0 \mathrm{~V}$ vs. RHE.

Reaction energies and barriers were calculated at various * $\mathrm{CO}$ coverages for all three facets. In general, the $\mathrm{CO}$ dimerization barrier becomes even more facile as the *CO coverage increases. Figure 4 illustrates this coverage effect on all three facets of $\mathrm{Cu}$. Increasing the coverage from $1 / 6 \mathrm{ML}$ to $2 / 3 \mathrm{ML}$ on (100) lowers the barrier from $0.45 \mathrm{eV}$ to $0.40 \mathrm{eV}$, a barrier reduction of $0.05 \mathrm{eV}$. Increasing the coverage from $1 / 6 \mathrm{ML}$ to $1 / 2$ ML on (111) lowers the barrier from $0.72 \mathrm{eV}$ to $0.40 \mathrm{eV}$, a barrier reduction of $0.32 \mathrm{eV}$. Increasing the coverage from 2/9 ML to 5/9 ML on (211) lowers the barrier from $0.72 \mathrm{eV}$ to $0.50 \mathrm{eV}$, a barrier reduction of $0.22 \mathrm{eV}$. The lowering of the barrier is attributed to adsorbate-adsorbate repulsion, which weakens the carbon bond to the surface and allows for more facile C-C bond formation. 


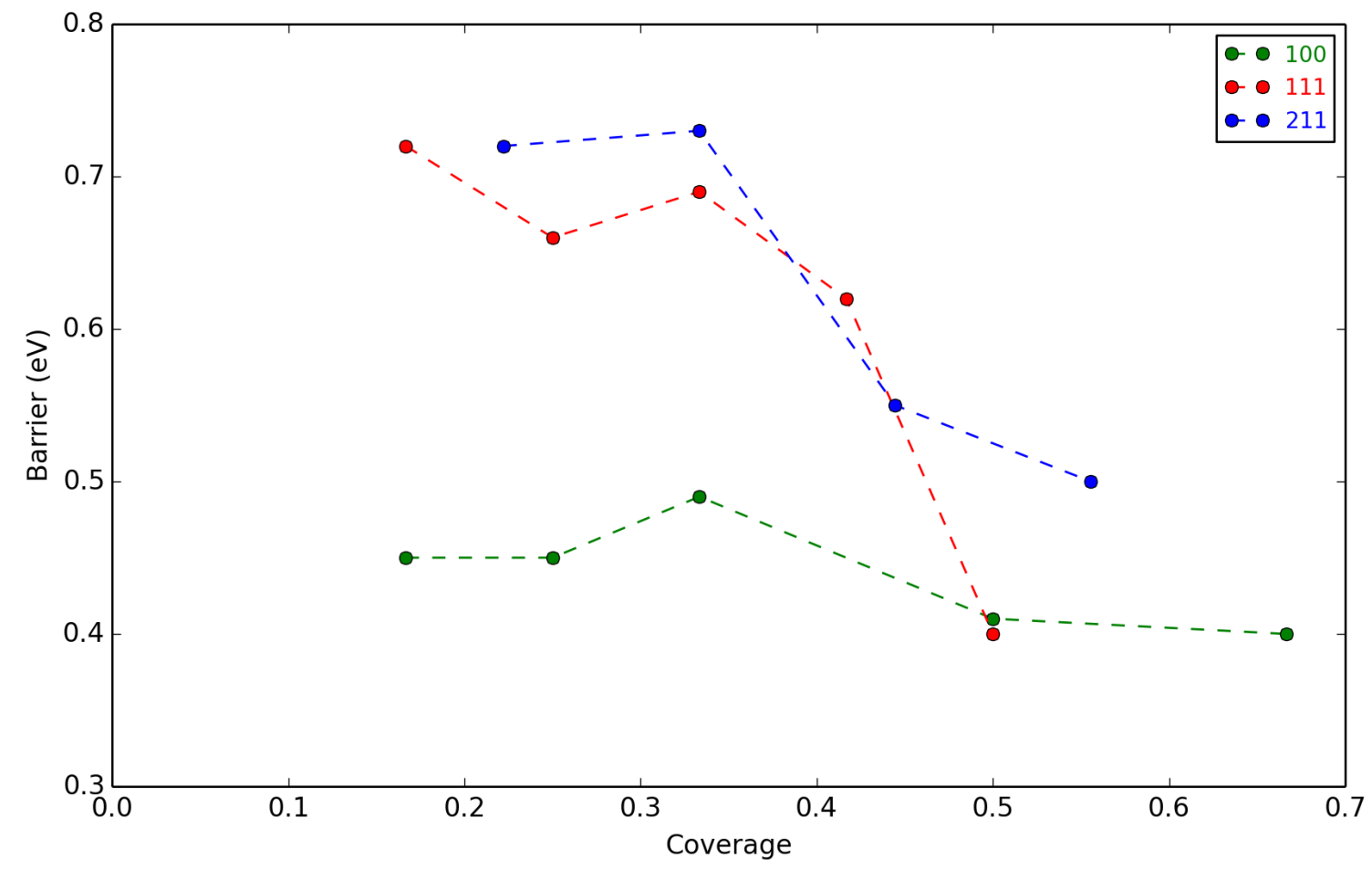

Figure 4. CO dimerization barrier as a function of coverage on all three facets. Dashed lines are included only to guide the eye.

\section{Strain effects}

Strain can allow for a further tuning of reaction energetics.[24] Experimentally this can be induced via overlayers, where the lattice mismatch between the overlayer and host causes the overlayer to become strained.[25] Strain has also been hypothesized to play an important role in $\mathrm{CO}_{2} \mathrm{RR}$ such as in the increase in activity on OD-Cu compared to polycrystalline $\mathrm{Cu} .[6]$

Figure 5 shows the energy of initial, transition, and final states at strains ranging from $-5 \%$ to $5 \%$ for the (100), (111), and (211) facets, and Figure 5 shows the corresponding free energy diagrams for $\mathrm{Cu}(100),(111)$, and (211) at strains of $-5,0$ and $5 \%$. Negative strain values indicate a decrease in the $x$ and $y$ distances in the bottom 2 fixed layers and vice versa for positive values. Overall, tensile strain decreases adsorption energies of all intermediates, and hence is expected to increase overall *CO coverage. For the case of (100), all states have very similar correlations with strain, as indicated by their similar slopes. However, for (111), the initial and final states have the same correlation but the transition state energies have a different slope. This results in a lowering of the barrier for tensile strains, with a $0.2 \mathrm{eV}$ decrease in the barrier for a 5\% strain. For (211), the initial and transition states have the same correlation but the final state energies have a different slope. This results in a decrease in the reaction energy for tensile strains. The trends in the energies are similar for (100) and (111), but are quite different for (211), which could arise from the additional degrees of freedom that the undercoordinated stepped sites on the (211) surface have to relax in response to strain. 
The free energy diagrams in Figure 6 exhibit these changes in barrier on (111) and reaction energy on (211).

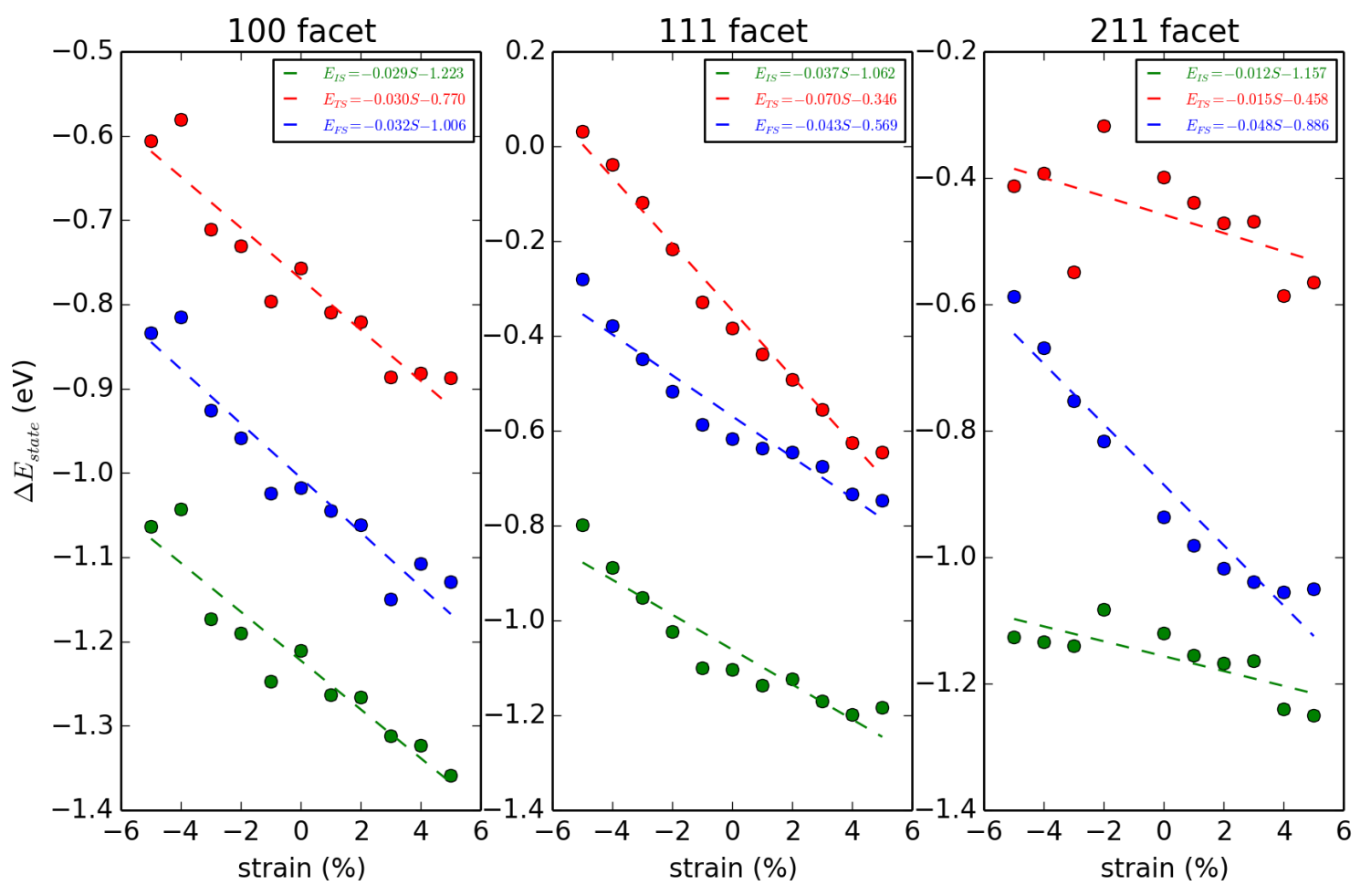

Figure 5. Energy vs strain for $\mathrm{Cu}(100),(111)$, and (211). 


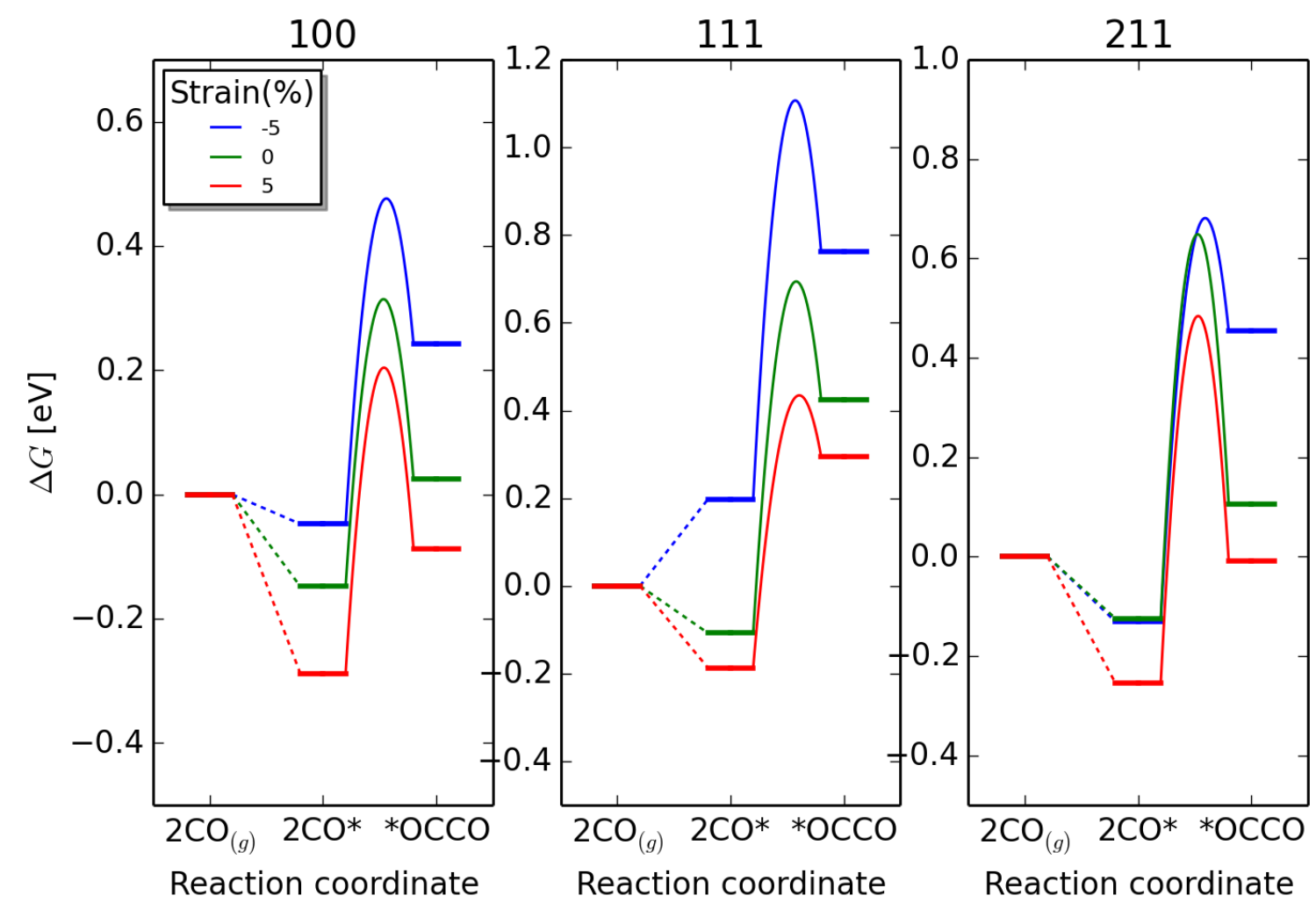

Figure 6. Free energy diagrams as a function of strain for $\mathrm{Cu}(100),(111)$, and (211).

The effect of strain can be attributed to both changes in electronic structure and coordination. Previous DFT calculations have shown *CO to bind stronger upon an increase in lattice constant on $\mathrm{Ru}(0001)$,[24] and this was also observed for * CO binding energies on all three $\mathrm{Cu}$ facets. For the (100) and (211) facets, there was no change in total coordination of the $2 * \mathrm{CO}$ to the surface between the initial and transition states (4 in initial, 4 in transition). However, for the (111) facet, there is a large change in total coordination between the initial and transition states (6 in initial, 3 in transition).

Overall, strain increases *CO coverage on all facets, and reduces the barrier on (111) and the reaction energy on (211). These results suggest that further improvement of $\mathrm{CO}$ dimerization rates on $\mathrm{Cu}$ facets is possible via a tensile strain.

Pourbaix analysis

In the presence of cations in the Helmholtz plane, CO-CO coupling is facile on all facets, and increased *CO coverage decreases the barrier further. Therefore, at absolute potentials negative of the $\mathrm{Cu}$ potential of zero charge (-0.7V vs. SHE),[14] CO dimerization may not be the limiting factor in the activity towards $\mathrm{C}_{2}$ products. Previous work on barriers for $\mathrm{CO}_{2} \mathrm{R}$ to $\mathrm{C}_{1}$ products have found proton-electron transfers to $\mathrm{C}$ to be generally difficult at low overpotential, while surface hydrogenations are generally facile, with the exception of $\mathrm{CO} \rightarrow \mathrm{CHO}$, which has a $\sim 0.9 \mathrm{eV}$ barrier on both $\mathrm{Cu}(111)$ and 
(211).[26] In the presence of a cation in the Helmholtz plane, it was found that OCCO* + $\mathrm{H}^{*} \rightarrow \mathrm{OCCHO}^{*}$ is also facile on (100) and (111),[11] with barriers that are only $\sim 0.05 \mathrm{eV}$ above the $\mathrm{CO}$ dimerization barriers. This suggests that $\mathrm{C}_{2}$ production at low overpotential may proceed primarily through CO dimerization and subsequent surface hydrogenation steps. Surface coverages of both *CO (which determines CO dimerization rate) and ${ }^{*} \mathrm{H}$ (which determines surface hydrogenation rate) would therefore be crucial in determining $\mathrm{C}_{2}$ activity.

We have explored surface coverages of $\mathrm{H}^{*}$, $\mathrm{CO}^{*}$, and $\mathrm{OH}^{*}$ under equilibrium conditions through Pourbaix diagrams.[27] Figures 7-9 show the Pourbaix diagrams for $\mathrm{Cu}$ (100), (111), and (211). Generally, as potential decreases, * $\mathrm{OH}$ is replaced by *CO, then ${ }^{*} \mathrm{CO}$ by $* \mathrm{H}$. At the onset potential for $\mathrm{CO}$ reduction to $\mathrm{C}_{2}$ products $(-0.3 \mathrm{~V}$ vs. RHE, $1 \mathrm{~atm} \mathrm{CO}$ )[6], $\theta_{\mathrm{CO}}=0.4$ on (100), $\mathrm{H}^{*}$ dominates the (111) facet, and on (211) $\theta_{\mathrm{CO}}=0.1$, $\theta_{\mathrm{H}}=0.3$. Therefore, while (100) has the lowest barrier and highest CO coverage, its activity towards $\mathrm{C}_{2}$ products may be limited by a low $\mathrm{H}^{*}$ coverage, which determines in part the surface hydrogenation rates. The (211) facet has a higher CO dimerization barrier, but it co-adsorbs significant coverages of both $* \mathrm{CO}$ and $* \mathrm{H}$, which would favor surface hydrogenation.

Striped regions in the diagrams indicate regions of $* \mathrm{CO}$ and $* \mathrm{H}$ co-adsorption, and point to the propensity of the (211) facet to co-adsorb ${ }^{*} \mathrm{CO}$ and $* \mathrm{H}$. Cu (100) and (111) contain narrow co-adsorption regions, around $-0.4 \mathrm{~V}$ and $-0.2 \mathrm{~V}$, respectively, while $\mathrm{Cu}$ (211) contains a large co-adsorption region that spans the potential range of $-0.6 \mathrm{~V}$ to $0.1 \mathrm{~V}$. We note that under $\mathrm{CO}_{2} / \mathrm{CO}$ reduction conditions, kinetics ultimately determine adsorbate coverage, but a thermodynamic analysis provides a first approximation of the propensity of the various facets to co-adsorb *CO and *H.

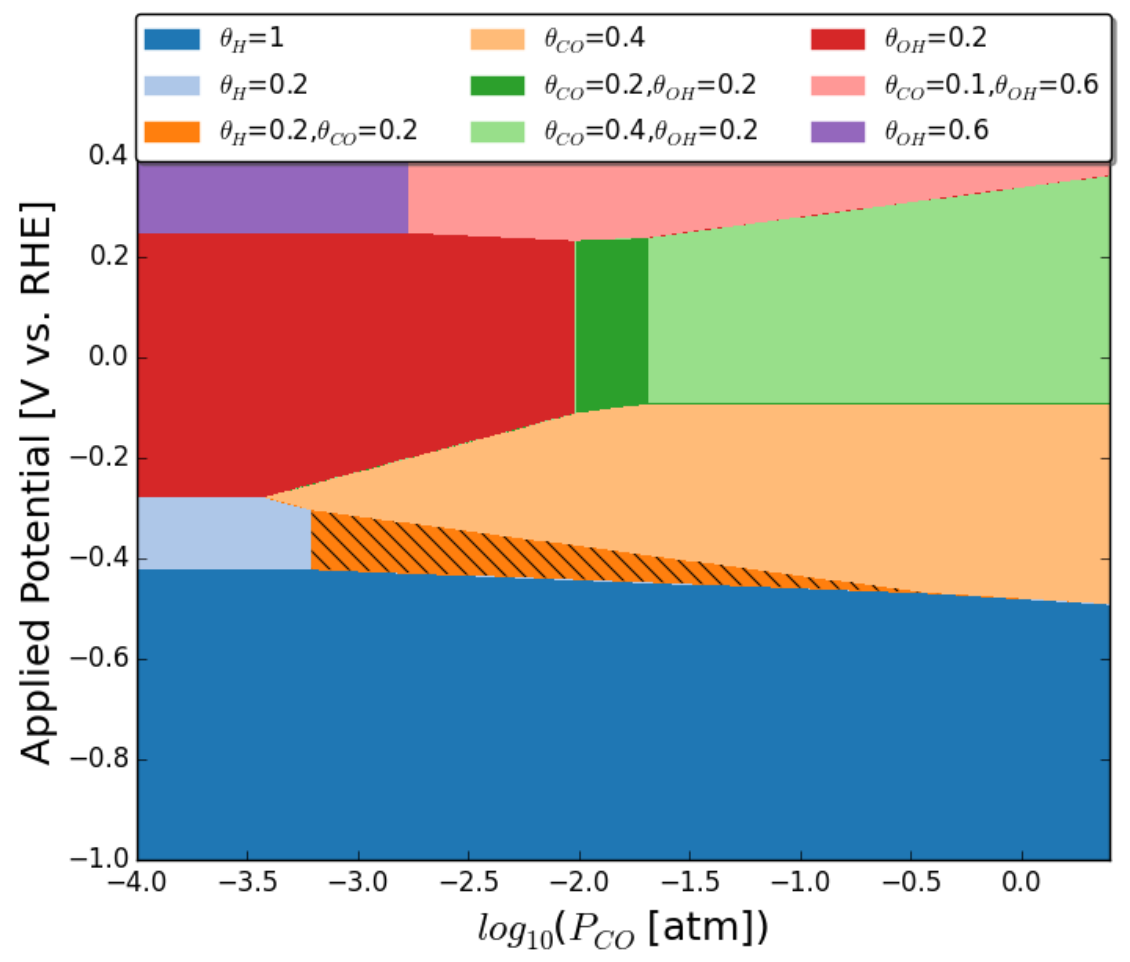


Figure 7. $\mathrm{Cu}(100)$ Pourbaix diagram.

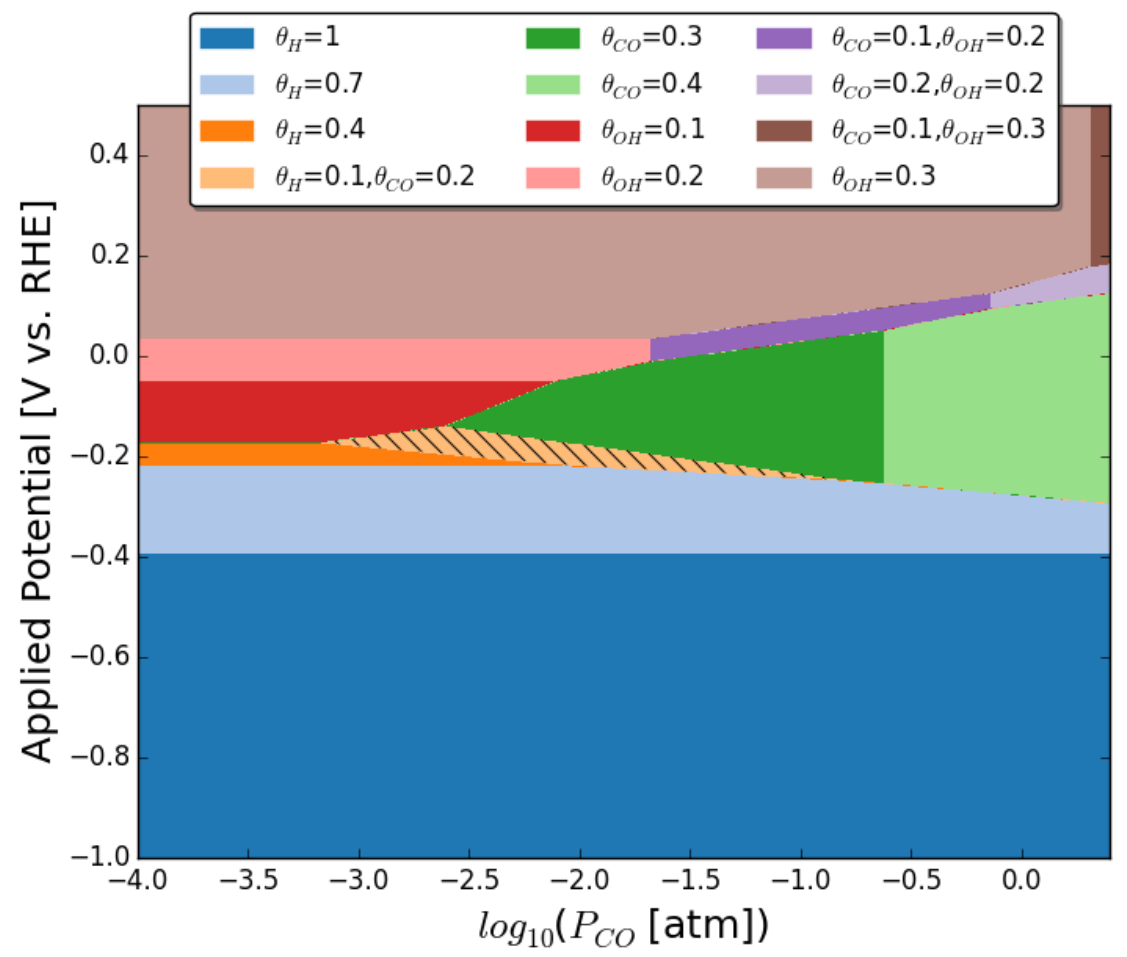

Figure 8. $\mathrm{Cu}(111)$ Pourbaix diagram.

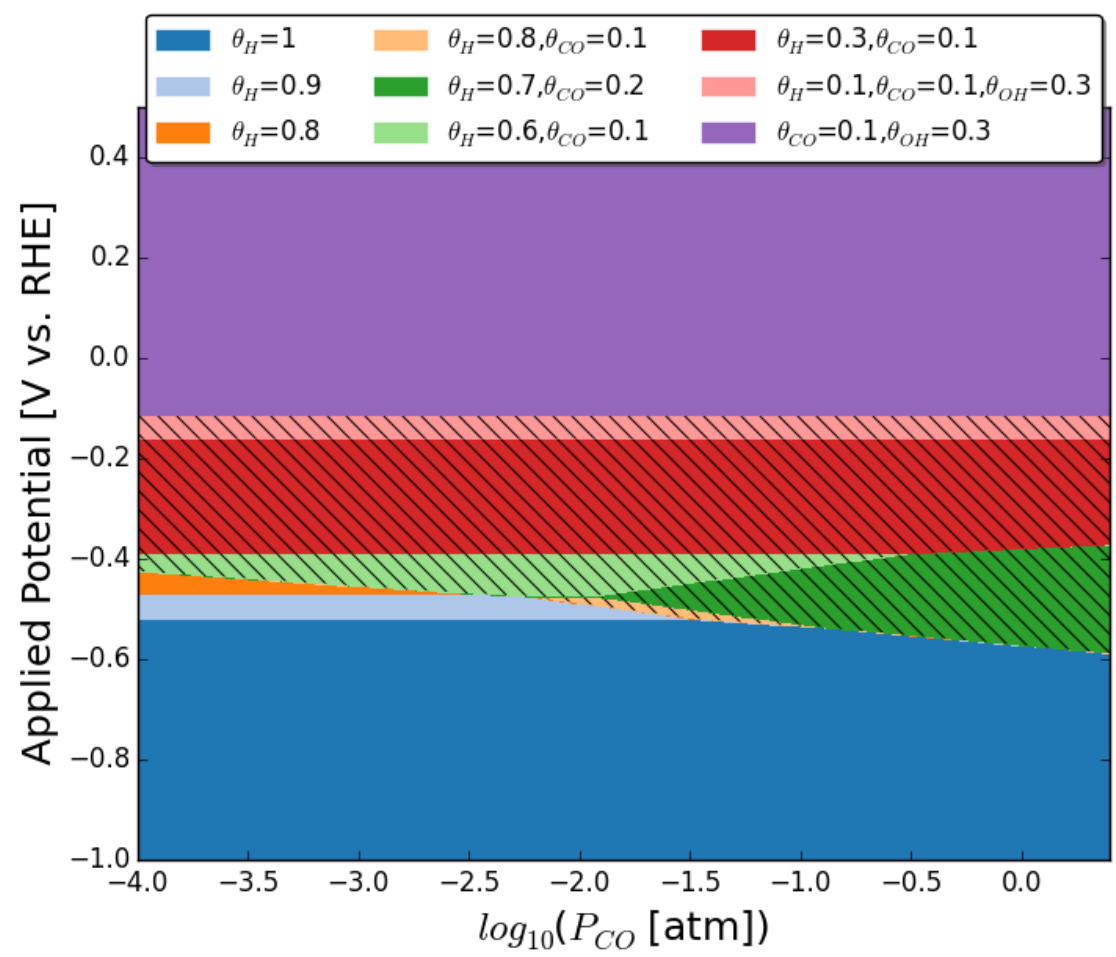

Figure 9. $\mathrm{Cu}(211)$ Pourbaix diagram.

\section{CONCLUSIONS}


We show CO dimerization to be feasible on basic facets of $\mathrm{Cu}$, namely (100), (111), and (211), in the presence of the field induced by cations at the electrochemical interface. All three facets have free energy barriers of $\sim 0.75 \mathrm{eV}$ or lower, indicating fast kinetics at room temperature. CO dimerization is therefore expected to play a role in $\mathrm{C}_{2}$ formation at potentials negative of the potential of zero charge of $\mathrm{Cu}$, corresponding to $\mathrm{CO}_{2} / \mathrm{CO}$ reduction in alkaline conditions. $\mathrm{Cu}(100)$ has the lowest $\mathrm{CO}$ dimerization barrier, but the stability of this surface remains controversial in the experimental literature.

When the coverage of ${ }^{*} \mathrm{CO}$ is increased on all three facets, the $\mathrm{CO}-\mathrm{CO}$ coupling energetics become even more facile, with barrier reductions ranging from $0.05 \mathrm{eV}$ to 0.32 $\mathrm{eV}$. Application of a tensile strain increases the ${ }^{*} \mathrm{CO}$ coverage, and reduces the barrier on (111) and reaction energy on (211). This suggests that tensile strain, realized through overlayers, can play a role in further improving *CO-*CO coupling energetics on $\mathrm{Cu}$.

Facile CO dimerization on all $\mathrm{Cu}$ facets suggest subsequent surface hydrogenation rates to play an important role in determining overall $\mathrm{C}_{2}$ activity. Surface hydrogenation requires the co-adsorption of $\mathrm{H}^{*}$. A Pourbaix analysis shows $\mathrm{Cu}(211)$ to have the largest propensity to co-adsorb ${ }^{*} \mathrm{CO}$ and $* \mathrm{H}$, which favors surface hydrogenation.

Future work will focus on the development of a microkinetic model of CO reduction, which will incorporate the reaction energetics, strain, coverage (via adsorbateadsorbate interaction models) and field effects discussed in this work. This kinetic model is expected to elucidate how the interplay of all such effects leads to overall activity and selectivity on the various $\mathrm{Cu}$ facets.

\section{METHODS}

Density Functional Theory (DFT) was used to calculate all electronic energies. Quantum Espresso was used with the BEEF-vdW exchange-correlation functional and Ultrasoft pseudopotentials. A $500 \mathrm{eV}$ plane-wave cutoff, $5000 \mathrm{eV}$ density cutoff, and a $(4,4,1)$ k-point sampling were used for all calculations. A $4 \times 3 \times 3$ supercell of fcc $\mathrm{Cu}$ (100) and (111) with a single layer of eight $(\mathrm{Cu}(100))$ or seven $(\mathrm{Cu}(111))$ water molecules at the surface was used to model the electrochemical interface. For the (211) surface, a $3 \times 3 \times 3$ supercell with five water molecules was used. Water densities were chosen to be as close as possible to that of the $\mathrm{Pt}(111)$ bilayer structure as found in UHV experiments within the unit cell sizes considered.[28] Water layer structures were determined using a minima-hopping algorithm that alternates between Molecular Dynamics and geometry optimization steps to construct a series of local minima.[29] All strain calculations were done by straining the initial and final states in both the $x$ and $y$ directions and then performing geometry optimizations to allow for relaxation in the $z$ direction. To simulate a charged double layer at the electrochemical interface, a single hydrogen atom was placed in the water layer. The ground-state electronic structure redistributes the charge from this atom's one electron to the metal, creating a chargeseparated double layer.[16] A dipole correction in the z-direction was applied in all calculations to compensate for the field resulting from the asymmetric slab in the vacuum region.[30] The correction to the *OCCO electronic energy and *OC-CO transition state energy was also taken from previous work.[11] 
The following elementary steps were considered to construct Pourbaix diagrams of $* \mathrm{H}$, ${ }^{*} \mathrm{OH}$, and ${ }^{*} \mathrm{CO}$ :

$$
\begin{aligned}
& H_{(a q)}^{+}+e^{-}+* \rightarrow H^{*} \\
& H_{2} O_{(l)}+{ }^{*} \rightarrow \mathrm{OH}^{*}+H_{(a q)}^{+}+e^{-} \\
& \mathrm{CO}_{(g)}+^{*} \rightarrow \mathrm{CO}^{*}
\end{aligned}
$$

The CHE model was used to equate the chemical potential of proton-electron pairs and hydrogen gas at standard conditions $\left(\mathrm{pH}=0, \mathrm{p}\left(\mathrm{H}_{2}\right)=1\right.$ bar $)$ and $\mathrm{U}=0 \mathrm{~V}$ vs SHE:

$$
H_{(a q)}^{+}+e^{-} \leftrightarrow \frac{1}{2} H_{2(g)}
$$

Binding energies were calculated based on these elementary steps. Free energy corrections were applied for the references $\left(\mathrm{CO}_{(\mathrm{g})}, \mathrm{H}_{2} \mathrm{O}_{(\mathrm{l})}, \mathrm{H}_{2(\mathrm{~g})}\right)$ and adsorbates $\left({ }^{*} \mathrm{CO}\right.$, $* \mathrm{OH},{ }^{*} \mathrm{H}$ ) to determine binding free energies. Because these calculations were done in vacuum, a solvation stabilization of $0.1 \mathrm{eV}$ was used for * $\mathrm{CO}$ and $0.5 \mathrm{eV}$ for $* \mathrm{OH}$ to simulate an electrochemical aqueous environment.[31] These solvation corrections were determined by adsorption calculations with and without a water layer on (111) facets of $\mathrm{Pt}($ for $* \mathrm{OH}$ ) and $\mathrm{Cu}$ (for $* \mathrm{CO}$ ). This $0.5 \mathrm{eV}$ stabilization of $* \mathrm{OH}$ was applied to a maximum of $3 \mathrm{OH}^{*}$, which represents the maximum coverage that can be hydrogenbonded in a hexagonal ice-like water structure. $\mathrm{Cu}$ (100), (111), and (211) were investigated by performing a series of calculations at mixed coverages of the three adsorbates. Using a $3 \times 3$ unit cell ( 9 sites) with three different adsorbates and a vacancy (4 possibilities), hundreds of mean-field mixed coverages are possible. A machine-

learning approach was used to identify energetically favorable coverages using only 50 DFT calculations. A training set of about 10-15 mixed coverages was chosen. The free energies of these coverages were calculated and the results used as inputs to a machinelearning package. The approach, based on Gaussian process regression, aims to predict the free energy of all possible mean-field adsorbate coverages for a finite surface.[32] It efficiently identifies the most stable configurations that form the lower hull of a 1-D Pourbaix diagram as a function of applied potential. With the DFT calculated energies, a 2-D Pourbaix diagram was determined for each $\mathrm{Cu}$ facet as a function of both applied potential and $\mathrm{CO}_{(\mathrm{g})}$ pressure (figures 7-9).

\section{ACKNOWLEDGEMENTS}

This material is based in part on work supported by the Air Force Office of Scientific Research through the MURI program under AFOSR Award No. FA9550-10-1-0572. This material is based in part upon work performed by the Joint Center for Artificial Photosynthesis, a DOE Energy Innovation Hub, supported through the Office of Science of the U.S. Department of Energy under Award Number DE-SC0004993. Discussions with Dr. Chuan Shi are gratefully acknowledged.

\section{REFERENCES}


[1] P.C.K. Vesborg, T.F. Jaramillo, Addressing the terawatt challenge: scalability in the supply of chemical elements for renewable energy, RSC Adv. 2 (2012) 7933. doi:10.1039/c2ra20839c.

[2] Y. Hori, Electrochemical CO 2 Reduction on Metal Electrodes, Mod. Asp. Electrochem. (2008) 89-189. doi:10.1007/978-0-387-49489-0_3.

[3] K.P. Kuhl, E.R. Cave, D.N. Abram, T.F. Jaramillo, New insights into the electrochemical reduction of carbon dioxide on metallic copper surfaces, Energy Environ. Sci. 5 (2012) 7050. doi:10.1039/c2ee21234j.

[4] J.H. Montoya, A. a. Peterson, J.K. Nørskov, Insights into C-C Coupling in CO2 Electroreduction on Copper Electrodes, ChemCatChem. 5 (2013) 737-742. doi:10.1002/cctc.201200564.

[5] K.J.P. Schouten, Z. Qin, E.P. Gallent, M.T.M. Koper, Two pathways for the formation of ethylene in CO reduction on single-crystal copper electrodes, J. Am. Chem. Soc. 134 (2012) 9864-9867. doi:10.1021/ja302668n.

[6] C.W. Li, J. Ciston, M.W. Kanan, Electroreduction of carbon monoxide to liquid fuel on oxide-derived nanocrystalline copper., Nature. 508 (2014) 504-7. doi:10.1038/nature13249.

[7] R. Kas, R. Kortlever, A. Milbrat, M.T.M. Koper, G. Mul, J. Baltrusaitis, Electrochemical CO2 reduction on Cu2O-derived copper nanoparticles: controlling the catalytic selectivity of hydrocarbons., Phys. Chem. Chem. Phys. 16 (2014) 12194-201. doi:10.1039/c4cp01520g.

[8] M. Gattrell, N. Gupta, a. Co, A review of the aqueous electrochemical reduction of CO2 to hydrocarbons at copper, J. Electroanal. Chem. 594 (2006) 1-19. doi:10.1016/j.jelechem.2006.05.013.

[9] K.J.P. Schouten, Y. Kwon, C.J.M. van der Ham, Z. Qin, M.T.M. Koper, A new mechanism for the selectivity to C1 and C2 species in the electrochemical reduction of carbon dioxide on copper electrodes, Chem. Sci. 2 (2011) 1902. doi:10.1039/c1sc00277e.

[10] F. Calle-Vallejo, M.T.M. Koper, Theoretical considerations on the electroreduction of CO to C2 Species on $\mathrm{Cu}(100)$ electrodes, Angew. Chemie - Int. Ed. 52 (2013) 7282-7285. doi:10.1002/anie.201301470.

[11] J.H. Montoya, C. Shi, K. Chan, J.K. Nørskov, Theoretical Insights into a CO Dimerization Mechanism in CO2 Electroreduction, J. Phys. Chem. Lett. (2015) 2032-2037. doi:10.1021/acs.jpclett.5b00722.

[12] A.S. Varela, M. Kroschel, T. Reier, P. Strasser, Controlling the selectivity of CO2 electroreduction on copper: The effect of the electrolyte concentration and the importance of the local pH, Catal. Today. 260 (2016) 8-13. doi:10.1016/j.cattod.2015.06.009. 
[13] B. Kim, S. Ma, H.R. Molly Jhong, P.J.A. Kenis, Influence of dilute feed and pH on electrochemical reduction of $\mathrm{CO} 2$ to $\mathrm{CO}$ on $\mathrm{Ag}$ in a continuous flow electrolyzer, Electrochim. Acta. 166 (2015) 271-276. doi:10.1016/j.electacta.2015.03.064.

[14] A. Łukomska, J. Sobkowski, Potential of zero charge of monocrystalline copper electrodes in perchlorate solutions, J. Electroanal. Chem. 567 (2004) 95-102. doi:10.1016/j.jelechem.2003.11.063.

[15] S. Trasatti, International Union of Pure and Applied Chemistry Commission on Electrochemistry * the Absolute Electrode Potential : an Explanatory Note, Pure Appl. Chem. 58 (1986) 955-966. doi:10.1351/pac198658070955.

[16] J. Rossmeisl, E. Skúlason, M.E. Björketun, V. Tripkovic, J.K. Nørskov, Modeling the electrified solid-liquid interface, Chem. Phys. Lett. 466 (2008) 68-71. doi:10.1016/j.cplett.2008.10.024.

[17] L.D. Chen, M. Urushihara, K. Chan, J.K. Nørskov, Electric Field Effects in Electrochemical CO2 Reduction, (unpublished).

[18] D.R. Strongin, G.A. Somorjai, The effects of potassium on ammonia synthesis over iron single-crystal surfaces., J. Catal. 109 (1988) 51-60.

[19] L. Vitos, A. V. Ruban, H.L. Skriver, J. Kollár, The surface energy of metals, Surf. Sci. 411 (1998) 186-202. doi:10.1016/S0039-6028(98)00363-X.

[20] H. Matsushima, A. Taranovskyy, C. Haak, Y. Gru, Reconstruction of Cu ( 100 ) Electrode Surfaces during Hydrogen Evolution, J. Am. Chem. Soc. (2009) 1036210363.

[21] Y. Kim, J.H. Baricuatro, A. Javier, J.M. Gregoire, M.P. Soriaga, The Evolution of the Polycrystalline Copper Surface, First to $\mathrm{Cu}(111)$ and Then to $\mathrm{Cu}(100)$, at a Fixed CO 2 RR Potential: A Study by Operando EC-STM, Langmuir. (2014) 15053-15056.

[22] A.C. Lausche, A.J. Medford, T.S. Khan, Y. Xu, T. Bligaard, F. Abild-Pedersen, et al., On the effect of coverage-dependent adsorbate-adsorbate interactions for CO methanation on transition metal surfaces, J. Catal. 307 (2013) 275-282. doi:10.1016/j.jcat.2013.08.002.

[23] C. Shi, H. a Hansen, A.C. Lausche, J.K. Nørskov, Trends in electrochemical CO2 reduction activity for open and close-packed metal surfaces., Phys. Chem. Chem. Phys. 16 (2014) 4720-7. doi:10.1039/c3cp54822h.

[24] M. Mavrikakis, B. Hammer, J. Nørskov, Effect of Strain on the Reactivity of Metal Surfaces, Phys. Rev. Lett. 81 (1998) 2819-2822. doi:10.1103/PhysRevLett.81.2819.

[25] L.A. Kibler, A.M. El-Aziz, D.M. Kolb, Electrochemical behaviour of pseudomorphic overlayers: Pd on Au(1 1 1), J. Mol. Catal. A Chem. 199 (2003) 57-63. doi:10.1016/S1381-1169(03)00018-9. 
[26] C. Shi, K. Chan, J.S. Yoo, J.K. Nørskov, Barriers of Electrochemical CO2 Reduction on Transition Metals, Org. Process Res. Dev. (2016) 1-22. doi:10.1021/acs.oprd.6b00103.

[27] J.K. Hansen, Heine A., Rossmeisl, Jan, Nørskov, Surface Pourbaix diagrams and oxygen reduction activity of Pt, Ag and Ni(111) surfaces studied by DFT, Phys. Chem. Chem. Phys. 140 (2008) 11-24; discussion 93-112. doi:10.1039/b812859f.

[28] H. Ogasawara, B. Brena, D. Nordlund, M. Nyberg, a Pelmenschikov, L.G.M. Pettersson, et al., Structure and bonding of water on Pt(111)., Phys. Rev. Lett. 89 (2002) 276102. doi:10.1103/PhysRevLett.89.276102.

[29] S. Goedecker, Minima hopping: An efficient search method for the global minimum of the potential energy surface of complex molecular systems, J. Chem. Phys. 120 (2004) 9911-9917. doi:10.1063/1.1724816.

[30] L. Bengtsson, Dipole correction for surface supercell calculations, Phys. Rev. B. 59 (1999) 12301-12304. doi:10.1103/PhysRevB.59.12301.

[31] A. a. Peterson, F. Abild-Pedersen, F. Studt, J. Rossmeisl, J.K. Nørskov, How copper catalyzes the electroreduction of carbon dioxide into hydrocarbon fuels, Energy Environ. Sci. 3 (2010) 1311. doi:10.1039/c0ee00071j.

[32] Z.W. Ulissi, A.R. Singh, C. Tsai, J.K. Nørskov, Automated Discovery and Construction of Surface Pourbaix Diagrams using Machine Learning, (in press). 